Théologiques

Théologiques

\title{
L'Église et la divination au Moyen Âge, ou les avatars d'une pastorale ambiguë
}

\section{Pierre Boglioni}

Volume 8, numéro 1, printemps 2000

La superstition

URI : https://id.erudit.org/iderudit/005001ar

DOI : https://doi.org/10.7202/005001ar

Aller au sommaire du numéro

\section{Éditeur(s)}

Faculté de théologie de l'Université de Montréal

\section{ISSN}

1188-7109 (imprimé)

1492-1413 (numérique)

Découvrir la revue

Citer cet article

Boglioni, P. (2000). L'Église et la divination au Moyen Âge, ou les avatars d'une pastorale ambiguë. Théologiques, 8(1), 37-66. https://doi.org/10.7202/005001ar
Résumé de l'article

Le christianisme condamna dès les premiers siècles, surtout après Saint-Augustin, toute forme de divination : l'oniromancie, la bibliomancie ettoutes autres techniques traditionnelles, qui semblaient trop entachées depaganisme. Dans la pratique, toutefois, l'Église fut beaucoup plus tolérante,lorsque ces mêmes pratiques étaient utilisées dans un contexte qu'elleconsidérait sans danger théologique ou pastoral. Le recours à la divinationdevenait ainsi un outil discrétionnaire de contrôle, notamment par rapportà la divination populaire. Par un choix très différent, l'Islam considéra lestechniques divinatoires comme une branche des sciences profanes, qui nemenaçaient en rien la religion et que ses fidèles pouvaient pratiquerlibrement. 


\title{
L'Église et la divination au Moyen Âge, ou les avatars d'une pastorale ambiguë
}

\author{
Pierre BOGLIONI \\ Département d'Histoire \\ Université de Montréal
}

\section{Introduction}

Une curiosité intense pour les choses à venir, et la recherche de " clés » pour en dévoiler à l'avance le secret, constituent l'une des composantes majeures de la culture médiévale. À des degrés et selon des modalités diverses, cette curiosité touchait tous les milieux, portait sur tous les domaines, utilisait toutes les techniques. Le savant scrutait le cours des étoiles pour en déduire le destin des individus et des nations. Le théologien scrutait les Écritures pour y décoder, cachées dans les replis de textes obscurs et de chiffres mystérieux, les dates du dernier drame du monde. ${ }^{1}$ Le paysan écoutait le chant du coucou le premier mai, pour savoir combien d'années il lui resterait à vivre. Partout circulaient des listes d'empereurs, de rois ou de papes à venir, signalant à l'avance leur caractère, leurs hauts faits et leurs déboires. ${ }^{2}$

1. "La pensée de l'Apocalypse escorte le Moyen Âge tout entier, non dans les replis de l'hérésie, dans le secret des petites sectes cachées, mais au grand jour et pour l'enseignement de tous " (Henri FOCILLON, L'An Mil, Paris, 1952, cité par M.-D. CHENU, Lumière et Vie 11 [sept. 1953], p. 105). Sur ces aspects, voir ma notice bibl. "Les millénarismes médiévaux. Aperçu de la recherche récente ». Dans Millénarismes. Au seuil de l' an 2000. Sous la dir. de Guy ROCHAIS [numéro spécial de Religiologiques. Sciences humaines et religion, 20 (automne 1999)], p. 87-111.

2. Marjorie REEVES, spécialiste de ce genre de littérature, considérait l'engouement pour les prophéties comme l'une des caractéristiques du Moyen Âge : "Perhaps we might say that only when intelligent and educated men ceased to take the prophecy seriously were the Middle Ages truly at an end » (Prophecy in the Later Middle Ages, p. 508). Sur les papes, les " prophéties de Malachie " (qui datent toutefois de c. 1590) sont probablement les plus célèbres. La liste comportait pour chaque pape, à partir de Célestin II (114344) jusqu'à un Pierre II qui serait le pape de la fin du monde, un bref motto pour décrire sa personnalité. 
En cela, le christianisme médiéval nous apparaît comme fort différent par rapport au christianisme post-tridentin. Malgré des condamnations théoriques répétées, il a toléré dans son sein des croyances et des attentes divinatoires, qu'il a marginalisées par la suite, et qui ne se retrouvent plus aujourd'hui que dans une culture folklorique évanescente, ou dans des pratiques individuelles et marginales. Face à ce constat, il se pose à l'historien un ensemble de problèmes en rapport avec toute la stratégie pastorale de l'Église par rapport aux substrats culturels des peuples qu'elle rencontrait. Déjà les cultures juive, grecque et romaine comportaient des systèmes complexes de croyances et de pratiques divinatoires. Mais l'Église allait rencontrer d'autres systèmes dans les cultures germanique et celtique. Des éléments tout aussi importants en subsistaient dans une culture paysanne omniprésente, remontant au néolithique. Qu'a-t-elle condamné et qu'a-t-elle toléré? A-t-elle seulement condamné et détruit, ou a-t-elle aussi accepté des compromis? Si tel est le cas, dans quels domaines et selon quels critères?

La réponse à ces questions n'est pas simple. D'abord, parce que le problème lui-même est d'une extraordinaire complexité. Les systèmes divinatoires se comptent par dizaines, chacun ayant une histoire propre. Que l'on songe à l'astrologie et à l'oniromancie, deux domaines auxquels se rapportent de vastes bibliographies. Plusieurs de ces pratiques par contre, surtout au niveau populaire, ne nous sont connues que de façon partielle, par des documents souvent inédits ou fort difficiles d'accès. Il manque encore un répertoire systématique des matériaux folkloriques du Moyen Âge. Il nous est impossible, par exemple, d'esquisser un tableau même approximatif de la divination par les animaux, alors qu'on sait, par des indices multiples, qu'elle a été fort variée et importante. ${ }^{3}$

3. Pour une approche générale, voir Divination, dans Encycoplaedia of Religion and Ethics, éd. J. HASTINGS, vol. IV (Édimbourg — New York, 1911), pp. 775-830 (avec une introd. générale et dix-sept notices spécifiques), et $\mathrm{La}$ divination. Études recueillies par André CAQUOT et Marcel LEIBOVICI, 2 vol., Paris, PUF, 1968, xx-358 et 580 p. (vingt-quatre articles, de grande qualité ; il y manque une étude sur la divination chrétienne). L'art. de T. ORTOLAN, Divination, dans le Dict. de théol. cath., IV/2 (Paris, 1911), col. 1441-1455, présente des matériaux juridiques et théologiques copieux, mais dans une perspective confessionnelle étroite. 
Deuxièmement, et surtout, parce que les historiens du christianisme se sont très peu intéressés au problème de l'acculturation réciproque du christianisme et des cultures ambiantes. Ils ont en général accepté une vision théologique et triomphaliste de la mission chrétienne, selon laquelle l'histoire du christianisme ne serait que la diffusion d'un message originel immuable, en rien entamé ou métamorphosé par les cultures des peuples qu'il a rencontrés. C'est une perspective qui ignore, ou camoufle sous la vague étiquette de "adaptations mineures", les changements substantiels que les croyances, l'imaginaire et les pratiques chrétiennes ont connu le long de l'histoire, sous la pression des substrats culturels que le christianisme a rencontrés. Les grandes absentes de l'historiographie du christianisme ont été et sont encore l'anthropologie et cette " religion populaire » qui en est le domaine de recherche le plus évident. ${ }^{4}$

Je ne saurais donc prétendre à une synthèse ou à un résumé systématique. Il ne me semble pas pertinent, non plus, de composer une typologie complète des moyens de divination, ou une casuistique des domaines et de situations, qui tournerait vite au catalogue. Je proposerai quelques esquisses sur des points privilégiés et quelques amorces de réflexion, dans la ligne des problèmes évoqués, pour prouver au moins l'intérêt historique et pastoral du thème de la divination dans l'Église médiévale. ${ }^{5}$

\section{Théologiens et pasteurs face à la divination}

L'ampleur et l'enracinement de la culture de la divination nous sont révélés par la constance et le sérieux avec lequel les théologiens

4. Cela vaut aussi pour la grande Kirchengeschichte publiée sous la direction de H. JEDIN (trad. angl. : Hubert JEDIN et John DOLAN, History of the Church, 10 vol., Londres, 1980-81), ainsi que pour la récente Histoire $d u$ christianisme (14 vol.), en cours de publication chez Desclée /Fayard.

5. L'art. de Jean-Claude SCHMITT, Les "superstitions ", dans J. LE GOFF et René RÉMOND, Histoire de la France religieuse, t. I (Paris, Seuil, 1988, pp. 416-551) constitue le meilleur cadre général. On y ajoutera, dans la bibl. plus accessible, Richard KIECKHEFER, Magic in the Middle Ages. Cambridge Univ. Press, 1989, 219 p. (surtout pp. 85-90 : "Divination and Popular Astrology »; pp.151-175) et Valerie I. J. FLINT. The Rise of Magic in Early Medieval Europe, Princeton Univ. Press, 1991, xii, 452 p. (passim). Mais voir aussi D. HARMENING, n. 7. 
ont dû s'en occuper, soit par des traités spécifiques, soit le plus souvent à l'intérieur même de leurs œuvres générales. La problématique de la divination apparaît comme incontournable pour le théologien médiéval, jusqu'à la Réforme incluse, tandis qu'elle n'occupe pratiquement aucune place dans la théologie actuelle.

Déjà Augustin, dans son traité Sur la doctrine chrétienne — rédigé pour l'essentiel en 396/7, comme une réflexion méthodologique sur ce qui, dans la culture païenne, pouvait être récupéré par un intellectuel chrétien - avait abordé avec une attention soutenue la culture divinatoire. Il avait proposé une liste succincte de ses principales techniques et de «ces milliers d'observances absolument stupides » (millia inanissimarum observationum), qui lui semblaient irrécupérables pour une culture chrétienne. ${ }^{6}$ Dans le traité Sur la divination des démons (c. 406) il allait parachever de démoniser la divination traditionnelle, en imputant aux pouvoirs extraordinaires des démons les extraordinaires réussites que l'opinion publique attribuait aux professionnels de la divination. Sur chacun de ces points, Augustin allait revenir maintes fois dans l'ensemble de son œuvre.

Au début du VII ${ }^{\mathrm{e}}$ siècle, Isidore de Séville allait relayer Augustin, dans ces Etymologies qui furent un des livres de départ de la culture médiévale. Il y donnait la liste d'une quinzaine de types de devins, avec une courte définition de leurs techniques et de leurs spécialités. Les magi étaient de redoutables généralistes, reconnus pour l'ampleur de leur pouvoir et la gravité de leurs crimes. Les necromanti avaient le pouvoir de «faire revenir les défunts pour répondre aux questions posées et révéler l'avenir ». Les hydromanti utilisaient de l'eau, parfois mêlée à du sang, "pour apercevoir en elle les images des démons et leur poser des questions ». Les divini prédisaient l'avenir par d'astucieuses conjectures. Les arioli pratiquaient la divination autour des autels et par des sacrifices funestes. Les haruspices déterminaient « certaines heures précises [à observer] pour la conduite des affaires » et inspectaient les entrailles des animaux pour prédire l'avenir. Les augures et les auspices

6. La démonisation de la divination y est déjà amorcée : "Toutes les techniques d'une telle superstition, sotte ou dangereuse, construites sur la base d'une communion pestifère entre les hommes et les démons, par une sorte de pacte d'amitié trompeuse et contraire à la foi, doivent être totalement repoussées par un chrétien »(II,36). 
étudiaient « le vol et les cris des oiseaux, ou d'autres signes soudains et imprévus ». Les phytonissae étaient des prophétesses inspirées directement par un dieu. Les astrologi tiraient leur divination des astres. Les genetliaci, appelés aussi vulgairement mathematici, prédisaient le destin de chacun en étudiant la position des étoiles au moment de sa naissance, tandis que les horoscopi prédisaient le destin en étudiant le moment même de la naissance. Les sortilegi étaient ceux qui, "sous couvert d'une fausse religion, pratiquent la divination par des sorts qu'on appelle sorts de saints ou par l'analyse d'autres écritures ». Les salisatores enfin prédisaient des événements heureux ou fâcheux "par les mouvements soudains du corps ».

D'un point de vue documentaire, cette liste théorique comportait des limites certaines. Elle était fondée plus sur le jeu des étymologies et sur les écrits d'Augustin que sur l'observation directe des réalités. Elle résumait la divination gréco-romaine, mais ignorait les formes nouvelles de la divination germanique. Elle visait des "professionnels » de la divination, mais ignorait les formes populaires et anonymes de celle-ci. Elle ne mentionnait pas l'oniromancie. Mais elle fut pour tout le Moyen Âge un outil pour conceptualiser et décrire la complexité de ce domaine. Il serait instructif de suivre en détail l'évolution de cette typologie tout au long du Moyen Âge. ${ }^{7}$ Les relais les plus importants seront le Des arts magiques par Raban Maur vers le milieu du IX ${ }^{\mathrm{e}}$ s., le traité Sur le divorce de Lothaire et Tetberge par Hincmar de Reims, une génération plus tard et, au début du $\mathrm{XI}^{\mathrm{e}}$ s., une section importante du Décret de Burchard de Worms. Au milieu du XII ${ }^{\mathrm{e}}$ s., à la croisée de la théologie, du droit et de la pastorale, le fondateur du droit canon, Gratien, réserva une place importante aux superstitions (en grande partie, la divination) dans la seconde partie de son Décret, à la Causa XXVI, organisée en sept sections, aux matériaux déjà fort riches et élaborés : Que sont les sortilèges et la divination? Quels sont les genres de la divination? Les jeteurs de sorts et devins doivent-ils être excommuniés?

7. Cela a été fait dans le travail indispensable de Dieter HARMENING, Superstitio. Überlieferungs und theoriegeschichtliche Untersuchungen zur kirchlich-theologischen Aberglaubensliteratur des Mittelalters, Berlin, 1979, 379 p. [bibl., p. 340-364]. 
Thomas d'Aquin lui-même, dans sa Somme de théologie, consacrera deux questions d'une ampleur substantielle à la superstition de la divination (II-II,q96) et à d'autres observances superstitieuses (IIII,q97), en mentionnant d'autres techniques en plus de celles évoquées par Isidore (par ex. : la chiromantia, la spatulimantia), et en isolant pour leur importance certains thèmes (astrologie, oniromancie, divination par les animaux, tirage au sort). S'il est vrai que ces deux questions (et leurs nombreux lieux parallèles) semblent elles aussi relever d'une culture livresque (notamment : Augustin, Isidore et Gratien), son opuscule Sur les sorts est une réponse à des questions concrètes qui lui avaient été posées. Tous les scolastiques allaient consacrer à ces thèmes un intérêt comparable à celui de Thomas d'Aquin.

Ce ne sont là que quelques repères, choisis dans divers domaines, de l'importance accordée par les théologiens au problème de la divination. Un enquête systématique dans le Nomenclator literarius theologiae catholicae par Hugo Huter relèverait des dizaines de traités de toute taille et de toute sorte. ${ }^{8}$ Encore lors des grands affrontements religieux de la Réforme, la théologien luthérien Kaspar Peucer (15251607) consacrait l'essentiel de son travail théologique à rédiger, en latin, un énorme Commentaire sur les principaux genres de divination, dans lequel ... les prophéties émises avec une autorité divine, et les prévisions scientifiques, sont distinguées des supercheries diaboliques et des observances superstitieuses ... (Wittemberg, 1553).

Une série parallèle de témoignages peut être cueillie dans la littérature pastorale. Ces témoignages ne sont pas aussi riches en descriptions détaillées que pourrait le souhaiter l'anthropologue moderne, mais ils n'en constituent pas moins, dans leur ensemble, un corpus d'une richesse remarquable : collections de sermons, textes synodaux ou conciliaires, pénitentiels, manuels des confesseurs, documents inquisitoriaux et textes narratifs de tout genre. ${ }^{9}$ Vers la fin du Moyen Âge, commença à émerger un filon de Traités sur les supersti-

8. Voir, à titre d'exemple, la notice sur Jean Gerson : De erroribus contra artem magicam (1421); De superstitiosa dierum observantia (1421); De observatione dierum quantum ad opera (1425); etc.

9. J'ai esquissé un inventaire de ces sources dans « Pour l'étude de la religion populaire au Moyen Âge : le problème des sources ", dans Foi populaire, foi savante. Paris, Éditions du Cerf, 1976, p. 93-148. Pour les sources du Haut Moyen Âge, voir aussi J. FLINT, The Rise of Magic, p. 36-58 ( "The Sources »). 
tions, qui développaient sous forme d'œuvres indépendantes la section sur les superstitions que les théologiens inséraient auparavant dans leurs sommes. ${ }^{10}$ Le plus connu de ces traités est celui de l'abbé Jean-Baptise Thiers, Traité des superstitions selon l'Écriture sainte, les décrets des conciles et les sentiments des saints Pères et des théologiens, qui date de la fin du XVII ${ }^{\mathrm{e}} .^{11}$

Dans toute cette documentation, la part faite à la divination est prépondérante, voire obsédante, surtout pour le Haut Moyen Âge. ${ }^{12}$ Il n'y a aucun autre thème qui, dans l'ensemble, revienne aussi fréquemment que la condamnation de la divination. Dès le Haut Moyen Âge, la terminologie qui désigne magiciens et devins s'est élargie à une cinquantaine de termes pour inclure des lemmes inconnus à la culture classique (tels que caragius), dont l'étymologie même est parfois difficile à déterminer. La typologie des moyens divinatoires s'est élargie, et comporte de nombreux cas qui sont des hapax, ou " cas uniques ", non signalés par aucun autre document : preuve indubitable de l'existence d'un substrat populaire complexe, qui arrivait difficilement à percer jusqu'au niveau du texte écrit. Certaines de ces techniques utilisaient des textes et des objets empruntés à la liturgie ou à d'autres aspects de la vie chrétienne.

Enfin, c'est par centaines que l'on peut compter les textes pastoraux officiels qui, dans les termes les plus solennels, interdisent l'accès à la divination $:^{13}$ "Si des hommes ou des femmes qu'on appelle

10. Ainsi, les deux questions de Thomas d'Aquin sur la divination font partie d'un bloc plus large de cinq questions portant sur la superstition (Somme de théologie, II-II, q. 92 à 96).

11. Une première édition ne comportait que les superstitions concernant les sacrements (Paris, A. Dezallier, 1659, 454 p.). Elle fut largement augmentée à 2 tomes en 4 vol. (I-II, A. Devillier, 1697 ; III-IV, J. De Nully, 1703-1704). L'éd. la plus courante est celle de Paris, La Compagnie des libraires, 1741, 4 vol in-12, que j'utilise ici.

12. Selon KIECKHEFER, " early medieval writers thought of magic primarily as a series of divinatory techniques... While other forms of magic attracted increasing attention in later medieval Europe, divination lost nothing of its appeal for the populace or its horror for moralists " (Magic in the Middle Ages, p. 85).

13. Bernadette FILOTAS, du Département d'histoire de l'Université de Montréal, achève une vaste thèse de doctorat sur Les survivances païennes 
devins, ou caragii ou lecteurs de sorts, sont invités dans la maison de quelqu'un (qu'ils soient goths, romains, syriens, grecs ou juifs) et si quelqu'un ose interroger l'avenir par leurs vains enchantements, qu'il soit suspendu de l'Église et qu'il paie au comte une amende de six onces d'or " (Concile de Narbonne, 589, c. 14) ; "Ceux qui recherchent les divinations et, à la façon des païens, leur donnent crédit, ou qui appellent des devins dans leur maison pour trouver quelque chose par la magie, ou pour contrer un mauvais présage, seront soumis à une pénitence de cinq ans " (Concile général de Paris, 829, c. 69); "Repoussez de vous et fuyez comme la peste les traditions mortelles des devins, diseurs de sorts et des charagi, qui ont commerce avec le diable » (Raban Maur, c. 850) ; "As-tu consulté les sorciers, les as-tu introduits chez toi pour rechercher un objet perdu ou pour faire des purifications? As-tu, selon les habitudes des païens, consulté des devins - tels que des prophètes - pour connaître l'avenir? As-tu consulté les jeteurs de sorts, les devins, les augures ou les enchanteurs? Si oui : deux ans de jeûne" (Corrector de Burchard de Worms, c. 69 ; c. 1010). Et, jusqu'à la fin du Moyen Âge, même le clergé se laissera tenter par ces pratiques, lorsqu'il n'en sera pas un agent direct : "Si un évêque, ou un prêtre, ou un diacre ou n'importe quel autre clerc est surpris à consulter des magiciens, des haruspices, des lecteurs de sorts ou d'autres qui exercent un art semblable, qu'il soit démis de sa charge et envoyé dans un monastère, pour une pénitence perpétuelle » (IV Concile de Tolède, a. 633, c. 29).

\section{Limite des résultats}

Ce barrage de défenses finit par avoir des effets marquants, qu'il importe de souligner pour donner au problème toute sa complexité. Une comparaison avec la divination dans la société musulmane du Moyen Âge est ici éclairante.

La recherche de T. Fahd, dont il n'existe pas l'équivalent pour le monde chrétien, a montré que l'Islam primitif a connu, comme le christianisme, le problème d'une riche culture divinatoire, qui trem-

dans la littérature pastorale du Haut Moyen Âge, dans laquelle elle enregistre systématiquement tous les textes et toutes les pratiques. Le chapitre le plus substantiel est consacré justement à " Magiciens et devins ". J'emprunte à cette recherche, que j'ai l'honneur de diriger, les matériaux cités ici. 
pait dans le culte païen et qui pouvait être considérée, à ce titre, comme réfractaire à la réforme monothéiste. Mais la solution de l'Islam fut très différente de la solution chrétienne : on bannit les devins ( kahins»), tout en valorisant les techniques divinatoires elles-mêmes, quitte à leur faire subir un processus de "laïcisation " qui les rendait inoffensives par rapport au système religieux. ${ }^{14} \mathrm{Je}$ citerai un long passage synthétique de l'auteur, qui résume bien l'essentiel de son analyse historique :

Bannir le devin de la société musulmane tout en conservant certaines pratiques divinatoires, cela nécessitait le réaménagement de ces dernières. Désormais, tout individu capte les signes divinatoires et les interprète. Il peut consulter quelqu'un de plus doué, plus capable, plus expérimenté que lui, sans que ce dernier ait une fonction officielle dans la communauté. Progressivement, et à la demande des califes qui se fiaient à la voix du ciel exprimée par les signes divinatoires, les héritiers de la science des devins, et non de leur fonction ni de leurs privilèges, fixent par écrit les codes d'interprétation et les règles des divers procédés divinatoires, de manière que chaque individu soit suffisamment armé pour donner aux signes qu'il retient la signification qu'il croit leur convenir. Il restera des personnes plus compétentes que d'autres; mais le principe de la «laïcité » de la divination n'en souffrira plus ... On ne considérait plus la connaissance divinatoire comme le fruit d'une inspiration divine, mais comme l'acquis d'une science humaine (p. 525).

Cette attitude permit l'éclosion d'une science divinatoire publiquement acceptée et honorée, comportant environ soixante techniques ou branches différentes, à la littérature élaborée (surtout pour l'oniromancie), qui constitua une tentation puissante pour les intellectuels chrétiens, notamment au XII ${ }^{\mathrm{e}}$ siècle, en pleine découverte de la science et de la philosophie arabes.

L'assaut de l'Église chrétienne contre la divination fut plus radical. Elle réussit à éliminer totalement les formes publiques et officialisées de la divination, telle que la prise d'auspices par les magistrats, ou l'interprétation et la conjuration publique des prodiges (procuratio prodigiorum) par un personnel spécialisé. Elle fit disparaître aussi les

14. Toufic FAHD, La divination arabe. Études religieuse, sociologique et folklorique sur le milieu natif de l'Islam. Leiden, E.J. Brill, 1966, xi-617 p., notamment la $\mathrm{II}^{\mathrm{e}}$ Partie : "Les procédés mantiques de l'Arabie ancienne et leur évolution dans l'Islam primitif » (p. 177-519). 
professionnels attitrés de la divination : le Moyen Âge chrétien ne reconnaîtra ouvertement ni la profession d'augure, ni celle d' " interprète des songes ", ni celle des pythonissae grecques et romaines, ni celle du file celtique, ni celle de la völva germanique. ${ }^{15} \mathrm{Il}$ est aussi remarquable que le christianisme n'ait jamais reconnu au clergé, à aucun niveau et sous aucune forme, un rôle stable et ritualisé de divination, analogue à celui qu'il lui a reconnu avec l'exorcisme dans les rapports avec le monde diabolique.

Finalement, et contrairement à la solution de l'Islam, il me semble que la pression de l'Église officielle ait obtenu des résultats stables dans deux directions majeures. D'un côté, elle a affaibli toutes les formes de divination artificielle ou opératoire, tout en étant un peu plus tolérante pour la divination intuitive, et surtout la divination clédonomastique ou par les signes. Ensuite, elle a réussi une marginalisation sociale de la divination et de ses agents. On constate que les interventions officielles contre la divination deviennent moins fréquentes et moins urgentes à partir du XIII ${ }^{\mathrm{e}}$ s., comme si la vitalité profonde de ces systèmes avait été tarie. Même chez les grands intellectuels laïcs (Dante, Boccace et Pétrarque dès le XIV s., puis la première génération d'humanistes au $\mathrm{XV}^{\mathrm{e}}$ ), on ne relève aucune curiosité particulière pour le monde de la divination, contrairement à ce qu'on pourrait relever chez des intellectuels musulmans de la même période et de niveau culturel comparable. Cette curiosité reviendra, sous la forme plus générale d'intérêt pour la magie, chez certains intellectuels de la Renaissance : Pic de la Mirandole, Jean Reuchlin, Cornelius Agrippa von Nettesheim, Théophraste Paracelse, Gerolamo Fracastoro, Gerolamo Cardano et d'autres. Mais, au même moment, l'émergence de la science à base mathématique et expérimentale condamnait ces mouvements à l'étiolement et à la marginalité culturelle.

Par contre, malgré les attaques constants de la part des pasteurs, la divination ne semble pas avoir faibli de façon substantielle chez le peuple en général, surtout à la campagne. Le retour des mêmes condamnations en serait déjà un preuve convaincante. Je relèverai toute-

15. Pour cette comparaison, voir, dans le recueil La divination, vol. I, les articles de Raymond BLOCH, "La divination en Étrurie et à Rome ", pp. 197-232 ; Françoise LEROUX, "La divination chez les Celtes », p. 233256, et R. DEROLEZ, « La divination chez les Germains », p. 257-302. 
fois, par quelques témoignages choisis, au moins deux points généraux. D'abord, le fait que les pratiques divinatoires étaient encore très variées et complexes à la fin du Moyen Âge. Ensuite, le fait que ces pratiques étaient enracinées et vivantes. Elles ne constituaient pas encore un "folklore" marginal et affaibli, mais une affirmation vigoureuse de croyances et de valeurs qui gardaient intacte leur force d'attraction.

Pour le premier point, je citerai d'abord les Évangiles des Quenouilles, une collection d'environ 230 croyances et pratiques populaires des régions de Flandres et Picardie, de la fin du XV ${ }^{\mathrm{e}}$ s. Ce document n'est pas un manuel de divination. Il s'agit plutôt d'un recueil générique, qui reflète la vie quotidienne d'un peuple de paysans. ${ }^{16}$ La perspective principale est pratique : la santé de la famille, la prospérité de la ferme, les soins des animaux. Puisque les narratrices sont des femmes, une place importante revient au mariage, aux conditions de sa réussite, aux moyens de s'assurer des enfants conformes à ses vœux. Or, dans cet ensemble générique, pas moins de quatrevingt croyances ou pratiques relèvent de la divination et des présages. Il se peut que l'auteur du recueil - un clerc cultivé, qui s'adressait à un public urbain - ait voulu recenser ces croyances dans une perspective d'ironie et de misogynie, mais cela même prouve le sérieux du système chez ceux qu'il voulait ainsi ridiculiser.

Le Traité des superstitions de J.-B. Thiers se situe chronologiquement au delà du Moyen Âge traditionnel, mais sa continuité culturelle avec les siècles précédents est évidente. La perspective est moins rurale que dans les Évangiles des Quenouilles et la charpente primitive de l'oeuvre, qui portait sur "les superstitions qui concernent les sacrements ", aurait dû en principe l'éloigner du monde de la divination. Pourtant, cette réalité occupe encore un place considérable dans le traité. Les livres $\mathrm{III}^{\mathrm{e}}$ et $\mathrm{IV}^{\mathrm{e}}$ du premier tome sont consacrés en entier à la divination (en sept chapitres) ${ }^{17}$ et à la " vaine observance » (en cinq

16. Les Évangiles des Quenouilles. Éd. critique, introd. et notes par Madeleine JEAY. Paris-Montréal, Vrin-PUM, 1985, 212 p., et Savoir faire. Une analyse des croyances des "Évangiles des Quenouilles» (XV ${ }^{e}$ s.). Montréal, Ceres, 1985, 311 p.

17. Dans l'édition de 1741, t. I, p. 174-263. Les principales subdivisions en sont : 1 . La divination en général ; 2 . La divination par les augures et les 
chapitres). ${ }^{18}$ Des centaines d'éléments sont ainsi recensés, selon les structures et pour les situations les plus diverses. Un autre « lieu» privilégié où sont enregistrées des pratiques multiples de divination et d'interprétation de signes est le traité sur le mariage : "Pour savoir si deux personnes seront mariées ensemble, si leur mariage prospérera, si elles y feront fortune, si elles auront de l'amitié l'une pour l'autre, si elles auront des enfants, si elles seront heureuses en enfants; à combien de vaines pratiques n'a-t-on point recours? ». ${ }^{19}$

Non seulement, donc, les pratiques condamnées depuis des siècles se sont maintenues pour l'essentiel, mais on s'aperçoit qu'elles ont évolué, que des pratiques nouvelles s'y sont ajoutées et que, désormais, c'est le christianisme même qui fournit la matière de la divination par ses sacrements, ses prières, ses objets du culte, ses dates sacrées : "Lorsqu'un garçon et une fille, un homme veuf et une femme veuve, tiennent un enfant, et qu'on veut savoir si le garçon et la fille, le veuf et la femme veuve, seront mariés ensemble, il faut observer si le cierge qu'on a allumé pour le Baptême de l'enfant, demeure allumé pendant toute la cérémonie, où s'il s'éteint ; s'il s'éteint, ni le garçon n'épousera la fille, ni l'homme veuf la femme veuve ; au lieu que s'il demeure allumé, le garçon sera marié à la fille, et l'homme veuf à la femme veuve $" .^{20}$ L'on décrit aussi une méthode de divination, sans doute moins populaire, que l'on pratiquait par les noms ou par les armes des cardinaux durant la vacance du Saint Siège! ${ }^{21}$ Presque tous ces matériaux auront encore une place importante, au XIX ${ }^{\mathrm{e}}$ siècle, dans le répertoire de De Chesnel. ${ }^{22}$

auspices (subdivisés en naturels et artificiels) ; 3. La divination par les événements et les rencontres ; 4 Formes diverses de divination artificielle ; 5. La divination par les rêves ; 6 . La divination par les sorts (distingués en : de partage ou division ; de consultation ; de divination) ; 7. La divination par l'astrologie. 18. T. I, p. 264-325. Les principales subdivisions en sont: 1 . La vaine observance en général ; 2 . L'art notoire ; 3 . Observance des jours, des mois, des temps et des années; 4. Observance des choses sacrées ou des reliques; 5 . Observance des santés. Une partie de cette matière relate des recettes efficaces, et non seulement divinatoires.

19. ch. III, p. 451. Voir aussi ch. III-V (t. IV, pp. 451-559).

20. T. IV, 1. X, ch. 1, p. 456.

21. T. I, l. III, ch. 4, p. 216.

22. A. De CHESNEL, Dictionnaire des superstitions, erreurs, préjugés et traditions populaires.... Paris, J.-P. Migne, 1856, 1360 coll. 
D'autres textes prouvent l'intensité avec laquelle le peuple restait attaché à ces pratiques, comme à une système culturel enraciné et difficile à détruire. Guillaume d'Auvergne témoigne d'une vigoureuse pratique de la divination et de l'interprétation des rêves dans un milieu populaire, encore au début du XIII ${ }^{\mathrm{e}}$ s., même si son interprétation de théologien le porte à considérer ces pratiques comme de la tricherie :

Quant aux divinations à manipuler ou des rêves à interpréter, ces vieilles femmes font preuve d'une telle avidité de mentir, et d'une si grande audace, que même avec le fouet on ne saurait les faire désister. Quant aux autres femmes en général, leur crédulité envers ces choses est tellement grande, qu'elles ne peuvent s'empêcher de fréquenter ces menteuses, même s'il est prouvé par de multiples expériences que ces vieilles devineresses se trompent, et ne devinent jamais la vérité. De plus, elles n'hésitent pas à payer un très haut prix pour tous ces mensonges et ces inventions. ${ }^{23}$

Presqu'un siècle plus tard, Humbert de Romans, en proposant aux prédicateurs dominicains un sermon à l'intention des "pauvres femmes dans les petits villages ", les met encore en garde contre l'attachement indéracinable de ces femmes envers les pratiques divinatoires :

Il y en a d'autres, qui se servent de ces divinations pour du profit, semblables à cette jeune fille devineresse qui faisait gagner beaucoup d'argent à ses maîtres (Actes 16, 15-18). D'autres sont d'une telle obstination, voire d'une telle incorrigibilité à l'égard de ces choses, qu'elles ne peuvent être détournées de cette (art de la) divination ni par des excommunications, ni par quelques menaces que ce soit. ${ }^{24}$

Je citerai enfin un exemplum bien connu par les prédicateurs du $\mathrm{XIII}^{\mathrm{e}}$ s. Ils racontent avec ironie l'histoire d'une vieille femme qui,

23. De universo, p. III, c. 27 (éd. Paris, 1674), p. 796b. Pour d'autres textes semblables voir mon art. "Peuple et culture populaire chez Guillaume d'Auvergne ", dans Mensch und Objekt im Mittelalter und in der frühen Neuzeit. Leben, Alltag. Kultur, Vienne, Österreich, Akademie der Wissenschaften, 1990, p. 193-222.

24. De modo prompte cudendi sermones circa omne hominum genus, Sermo 99, dans Bibliotheca maxima veterum patrum, Lyon, 1677, vol. XXV, p. 505. L'oeuvre date des années 1270-1274. 
ayant entendu un premier jour de mai le coucou chanter cinq fois, en avait conclu comme chose certaine qu'il lui restait encore cinq années à vivre. Étant tombée malade, et proche de mourir, sa fille l'exhortait à se confesser : mais elle répondit que cela n'était pas nécessaire, puisqu'elle avait encore cinq années à vivre. Lorsqu'elle eut perdu la parole, et on la poussait à se confesser, elle murmura cinq fois coucou. Bientôt, tout à fait incapable de parler, elle put juste lever les cinq doigts de la main, et c'est ainsi qu'elle expira. ${ }^{25}$

L'ironie du théologien est peut-être justifiée. Mais l'anthropologue relèvera le caractère tenace de cette croyance dans le pouvoir divinatoire des oiseaux, une croyance qui résiste même à l'épreuve des faits.

\section{Les raisons de la persistance}

Quelles sont les raisons de cette persistance tenace? Je crois qu'il faut chercher la réponse dans des lignes multiples et complémentaires, qui mériteraient d'être élaborées avec des concepts appropriés de psychologie sociale et d'anthropologie culturelle.

La première raison réside probablement dans l'urgence des problèmes que les systèmes divinatoires cherchaient à résoudre. Nous ne pouvons même plus imaginer, sans un effort que seule l'anthropologie peut soutenir et guider, le degré d'incertitude, d'angoisse, d'impuissance dans lequel vivaient les sociétés traditionnelles. Retrouver le bétail égaré, connaître l'issue d'un long voyage (surtout un voyage en mer) ou d'une maladie grave, pour citer des cas où le recours à la divination était des plus constants, étaient des situations existentielles décisives, chargées d'une insoutenable incertitude. La divination constituait une forme de réponse " rationnelle ", cautionnée qu'elle était par la culture ambiante. Elle est à interpréter selon les mêmes paramètres que la croyance indestructible aux miracles, qui constitue elle aussi une réponse collective à l'insoutenable angoisse de l'impuissance.

La deuxième raison serait à rechercher dans le fait que l'Église et ses pasteurs se sont limités, pour le fond, à une action répressive et négative et ne se sont pas montrés intéressés à créer un système officiel

25. ÉTIENNE DE BOURBON, Anectodes historiques..., éd. Lecoy de la Marche, n. 52, p. 59-60. L'épisode est connu aussi par Jacques de Vitry et Jourdain de Saxe. 
ou semi-officiel de divination, par lequel le simple fidèle aurait pu avoir recours (par le clergé, si nécessaire) à des moyens ouverts, acceptés et reconnus. En cela, la comparaison avec le traitement de la divination dans l'Islam est frappante. Le christianisme officiel aurait pu créer ce système en ouvrant un nouveau secteur, spécifique aux recours divinatoires, dans le monde déjà extraordinairement varié des rituels. L'hypothèse n'est pas historiquement invraisemblable. Le grand recueil de A. Franz nous permet de saisir l'extraordinaire richesse et variété de ce domaine, avant l'uniformisation imposée par Rome au XVIIe s. ${ }^{26}$ Dans son introduction méthodologique, l'auteur évoque les connivences multiples de cette littérature avec la culture ambiante, ainsi que l'origine d'en bas, de maints rites et formules. ${ }^{27}$ Rien de comparable ne fut fait pour la divination. En laissant ses fidèles sans alternatives véritables, l'Église les a en quelque sorte obligés à recourir aux systèmes défendus.

Une dernière raison est à chercher dans le fait que l'Église et ses intellectuels ne se sont jamais décidés à reconnaître — d'une façon claire, nettement théorisée, universellement valable - le caractère impénétrable du temps à venir, son altérité épistémique par rapport au temps présent, ainsi que la nature radicalement insondable de la Providence. Au contraire, théologie et pastorale ont toujours jonglé avec l'idée que, par certaines personnes et dans certaines circonstances et à certaines conditions, le voile du futur pouvait être levé. En ouvrant des brèches de toutes sortes dans son système de négation, l'Église a implicitement autorisé ses fidèles à en faire autant. Il est temps d'évoquer certaines de ces brèches.

\section{La pratique des " sorts bibliques"}

Nulle part l'attitude ambivalente de l'église médiévale face à la divination ne se révèle aussi clairement que dans l'histoire des sorts bibliques ou consultation des Évangiles. Cette pratique —à ne pas confondre avec

26. Die Kirchlichen Benediktionen im Mittelalter, 2 vol., Fribourg/B., 1909, XXXVIII-646 et VIII-764 p. (réimpr. Graz 1960).

27. "Le développement des usages liturgiques a reçu dans ce secteur son impulsion non d'en haut, mais d'en bas... C'est justement dans le domaine des sacramentaux que le génie populaire avec ses traits particuliers, et la culture du peuple dans ses états successifs, se sont imposés » (I, p. 13). 
celle des «sorts des apôtres » (sortes apostolorum), une technique plus complexe et qui eut une histoire très différente - consistait à ouvrir au hasard la Bible, pour y lire le premier passage qui tombait sous les yeux : ce passage avait valeur prophétique. Cette forme de divination était une adaptation chrétienne d'une pratique païenne analogue, qui attribuait le même rôle aux textes d'Homère chez les Grecs, ou de Virgile chez les latins. De nombreuses autres cultures la connaissent également. ${ }^{28}$

L'ambiguïté éclate déjà chez saint Augustin. Il écrivait à Januarius : "À propos de ceux qui interrogent le sort par les pages des évangiles, même s'il est préférable qu'ils fassent cela plutôt que de recourir aux démons, je dois avouer que cette pratique ne me plaît pas, à savoir de détourner les oracles qui parlent de choses divines à l'usage des affaires profanes et des choses de cette vie passagère $" .^{29}$ Mais il avouait en même temps, dans une page fameuse des Confessions (VIII,12), que le tournant décisif de sa vie avait eu lieu par l'ouverture au hasard du "livre de l'Apôtre ", quand ses yeux était tombés sur le passage : «point de ripailles ni d'orgies, pas de luxure ni de débauche » (Rom. 13,13). ${ }^{30}$

L'opinion négative d'Augustin fut confirmée par des condamnations ultérieures. Un capitulaire carolingien de 789 ordonnait : "que personne ne présume de lire les sorts dans le psautier, les Évangiles ou d'autres objets. » ${ }^{31}$ Le Pénitentiel d'Aroundel (c. 1000) condamnait à quarante jours de pénitence « ceux qui osent faire des divinations ou

28. Voir H. LECLERCQ, art. Sortes sanctorum, dans Dict. d'archéol. chrét. et de liturgie, XV/2, Paris, 1953, pp. 1590-1592 et surtout J.-Cl. POULIN, " Entre magie et religion. Recherches sur les utilisations marginales de l'écrit dans la culture populaire du Haut Moyen Âge ", dans P. BOGLIONI (dir.), La culture populaire au Moyen Âge. Montréal, L'Aurore, 1979, p. 121-143 (notamment pp. 130-136).

29. Ep. LV, Ad inquisitiones Januarii, I8.37 (PL 33, 222).

30. Cet épisode, introduit par un "oracle clédonomastique " typique (Augustin entend par hasard des voix d'enfants qui chantent : "Prends et lis »), a été commenté par Pierre LABRIOLLE, "Source chrétienne et allusions païennes de l'épisode du "Tolle, lege" ", Revue d'histoire et de philosophie religieuse, 32 (1952), 170-200 ; voir aussi P. COURCELLE, "L'enfant et les "sorts bibliques" ", Vigiliae christianae, 7 (1953), 194-220. 31. Duplex legationis edictum, 20 (Monum. Germaniae historica, Capit. Reg, Franc. 1, p. 64). Le texte sera repris dans leurs collections canoniques 
chercher des sorts dans le saint Évangile, ou dans les autres livres, ou par n'importe quel objet sacré ». Mais ces réticences et ces défenses n'ont pas empêché cette pratique d'être largement répandue. On s'en servait pour justifier ou confirmer le choix d'un évêque, pour obtenir un pronostic sur le mandat d'un prélat, ou pour toute autre affaire importante. Dans l'hagiographie du haut Moyen Âge, et dans d'autres sources narratives, on en trouve des dizaines de témoignages, qui couvrent une vaste typologie de situations.

La même pratique sera encore familière à François d'Assise, et sera enregistrée par ses biographes officiels, non sans de multiples précautions. Il l'utilisa à propos de la vocation de Bernard d'Assise :

« Demain, dès le matin, nous entrerons à l'église, nous prendrons le Livre des Évangiles, et c'est au Christ que nous demanderons conseil. » Ils entrèrent donc dans une église le lendemain matin, commencèrent par prier avec ferveur, puis ouvrirent l'Évangéliaire, prêts à accomplir tout ce que leur désignerait le premier passage rencontré. Ce fut le conseil donné par le Christ : "Si tu veux être parfait, va, vends tout ce que tu possèdes, et donnes-en le prix aux pauvres. » Ils tentèrent une deuxième fois et lurent : "Pour la route, n'emportez rien. » Une troisième expérience donna : "Celui qui veut venir après moi, qu'il se renonce à soimême. " Sans différer, Bernard réalisa tout ce programme et n'en transgressa pas un iota. ${ }^{32}$

François eut encore recours aux "sorts évangéliques " lorsque à la fin de sa vie, retiré à l'Alverne, il s'interrogeait sur son avenir et celui de son Ordre :

Il s'en alla prendre un jour l'Évangéliaire... et il supplia Dieu d'indiquer, par la première page où le volume s'ouvrirait, ce qu'il fallait faire pour couronner l'œuvre jadis commencée... Et voilà que le premier passage sur lequel il tomba était le récit de la passion de Notre-Seigneur JésusChrist. C'était révélé assez clairement qu'il aurait à souffrir. Mais, pour qu'on ne puisse mettre cette indication sur le compte du hasard, il ouvrit

par Réginon de Prüm (vers 906) et Burchard de Worms (v.1010). On trouvait une défense analogue dans l'Homilia de sacrilegiis, 8 (éd. CASPARI, p. 7), qui date de la fin du VIII ${ }^{\mathrm{e}}$ s.

32. Thomas de Celano, Vita secunda, 15 ; trad. D. VORREUX, Saint François d'Assise. Documents, Paris, 1968, p. 359. L'épisode est repris avec un grand relief par Bonaventure, Legenda Major 3,3 (ibidem, p. 602). 
le livre une deuxième fois, puis une troisième fois, et il trouva le même texte ou un texte équivalent. ${ }^{33}$

Mais ces mêmes témoignages, si vifs et immédiats, constituent une preuve supplémentaire de l'ambiguïté pastorale de la pratique. Il est évident en effet que Thomas de Celano ressent le besoin de justifier l'agir de François (qui était un simple laïc, non un théologien), en rappelant les exemples hagiographiques antérieurs ("son intention était celle-même des saints et des parfaits qui ont agi de la même façon »), et en soulignant que toute l'opération était accompagnée de prières intenses, "sous l'Esprit de Dieu, pour qu'on ne puisse mettre cette indication sur le compte du hasard ».

Même ces précautions rédactionnelles, toutefois, ne suffisaient pas à enlever tous les doutes sur la nature de cet épisode. Thomas de Celano le laissa tomber dans sa Vita secunda, soucieuse d'atténuer tout ce qui, chez François, pouvait prêter à critique. Bonaventure, qui le garde dans sa Legenda major, en réduit beaucoup la place, et se soucie de "théologiser » et de "dé-personnaliser » davantage l'épisode. C'est Dieu lui-même qui demande à François d'ouvrir les Évangiles, et c'est un compagnon de François, "frère vraiment pieux et saint ", qui accomplit la consultation. ${ }^{34}$ Dans les deux récits, la triple répétition de la consultation, qui n'a probablement qu'une portée folklorique (la triple répétition est une condition courante des épreuves dans le folklore) reçoit une interprétation théologique ("à cause de sa dévotion à la Trinité »). On peut penser que cette pratique était courante chez François, et que son souvenir était important dans la tradition de l'Ordre, mais que les deux biographes n'en ont pas transmis le souvenir sans réticences et maintes précautions.

33. Thomas de Celano, Vita Prima, II, 92-93 ; trad. D. VORREUX, p. 297. 34. "Or, Dieu parlant à son âme lui révéla qu'en ouvrant le livre des Évangiles il apprendrait du Christ ce qui allait être, en lui et par lui, le plus agréable à Dieu. Après une fervente prière, il alla prendre sur l'autel le livre des saints Évangiles. Et par trois fois, au nom de la sainte Trinité, le fit ouvrir par son compagnon, frère vraiment pieux et saint. Par trois fois on tomba sur le récit de la Passion du Sauveur " (Legenda Major, 13,2 ; trad. VORREUX, p. 702). 


\section{Le rêve divinatoire dans l'hagiographie}

La valeur divinatoire des rêves est un autre domaine dans lequel la pastorale et la pratique du christianisme médiéval ont manifesté une ambivalence certaine. En ligne de principe, il aurait semblé naturel de développer une oniromancie chrétienne, puisque la Bible comporte des exemples nombreux de songes inspirés par Dieu, aussi bien dans l'Ancien que le Nouveau Testament. ${ }^{35}$ Dans l'histoire de Joseph et de ses frères, la Bible latine utilise le terme somniator pour désigner Joseph, à savoir le même terme que les textes médiévaux utiliseront pour désigner l' "interprète des rêves ". ${ }^{36}$ Dans certains écrits de la première littérature chrétienne, comme dans l'extraordinaire Passion de Perpétue et Félicité, l'élément onirico-visionnaire joue un rôle primordial. ${ }^{37}$ La recherche de Martine Dulaey montre de façon captivante la multiple présence du rêve dans la biographie et la pensée d'Augustin et de son milieu. ${ }^{38}$ On pourrait tracer un tableau analogue à la fin du VI ${ }^{\mathrm{e}}$ s. d'après les Dialogues du pape Grégoire le Grand, notamment dans ce IV ${ }^{\mathrm{e}}$ livre qui concerne l'au-delà.

Par contre, d'autres textes de l'Écriture interdisaient l'accès aux oniromanciens. ${ }^{39}$ De plus, l'utilisation divinatoire des rêves était compromise, aux yeux des pasteurs chrétiens, par l'importance de l'oniromancie dans la tradition païenne, aussi bien populaire que savante. Toute l'oeuvre de Lucien de Samosate nous confirme, avec les romans populaires grecs et latins, le rôle des rêves dans la vie quotidienne du

35. L'Islam a développé un extraordinaire oniromancie, accessible à tous ses fidèles : voir FAHD, La divination arabe, p. 247-367 : «Les procédés oniromantiques ".

36. "Ils se dirent entre eux : Voilà l'homme aux songes [somniator] qui arrive " (Gen. 37,19).

37. Discuté dans E.R. DODDS, Pagan and christian in an age of anxiety, New York, The Norton Library, 1965, p. 47-53. Pour la Grèce antique, il avait discuté les rêves dans Les Grecs et l'irrationnel, Paris, Flammarion, 1977, p. 107-137.

38. Martine DULAEY, Le rêve dans la vie et la pensée de saint Augustin, Paris, Éditions Augustiniennes, 1973, 263 pp. (bibl., p. 253-259).

39. Ainsi : "Tu n'écouteras pas les paroles de ce prophète ni les songes de ce songeur (somniatoris) » (Deut. 13,3); "N'écoutez pas leurs songes, fruits de leurs rêves" (Jér. 29.8). Voir une liste des rêves bibliques et des textes bibliques sur les rêves dans M. DULAEY, Le rêve, p. 231-233. 
peuple et des classes moyennes. La philosophie néo-platonicienne renforça les croyances populaires, en proposant les rêves comme l'un des moyens privilégiés pour communiquer avec la divinité. Des textes hautement structurés, comme l'Interprétation des rêves par Artémidore de Daldis ( $\mathrm{II}^{\mathrm{e}} \mathrm{s}$. après J.-C.) et le Commentaire sur le songe de Scipion par Macrobe (fin du IV s.) assuraient une caution de prestige à une vaste littérature mineure et à l'activité des somniarii ou " interprètes des rêves ".

Le choix pastoral de l'Église penchera donc très tôt vers la méfiance. Déjà en 314 le concile d'Ancire interdit aux chrétiens tout usage de l'oniromancie. L'interdiction sera répétée maintes fois dans le christianisme antique. Au début du Moyen Âge, cette interdiction semble disparaître pendant deux siècles. Mais elle revient avec force, à partir du début du IX ${ }^{\mathrm{e}}$ s. : Concile de Paris (829), Capitula de Hérard de Tours (858), puis quelque deux douzaines d'autres textes, qui condamnent les somniatorum coniectores et les somniarii, à côté d'autres professionnels de la divination. D'après ces textes, il apparaît que la clientèle des "interprètes des rêves » était constituée aussi de clercs et autres membres du clergé : "Si un prêtre ou un clerc prête attention aux augures, aux divinations, aux rêves, aux sorts et aux phylactères ou petits feuillets écrits, qu'il soit expulsé du clergé. S'il s'agit d'un laïc, qu'il fasse pénitence pendant cinq ans " (Pénitentiel de Théodore, I, 15,4 c. 668-756, et Statuts de Boniface, 20, c. 930). Cette suite d'interdits empêchaient les chrétiens ordinaires d'avoir accès à leurs propres rêves et à leur signification. ${ }^{40}$

Mais en même temps, une réflexion théologique s'efforçait d'identifier les conditions des " bons " rêves venant de Dieu, opposés aux mauvais rêves venant du diable ou de l'homme lui même. Un système moral d'interprétation remplaçait ainsi un système technique, tandis que ces « bons » rêves devenaient l'apanage des bons rois, des grands personnages aux fonctions providentielles, et surtout des saints. ${ }^{41}$ Les

40. Voir Jacques LE GOFF, "Le christianisme et les rêves (II - VII s.) ", dans I sogni nel medioevo. A cura di Tullio GREGORY. Rome, Edizioni dell'Ateneo, 1983, p. 171-218, qui étudie dans une synthèse remarquable ces incertitudes et ce changement.

41. De nombreux récits de rêves sont recensés dans la synthèse de Maria Elisabeth WITTMER-BUTSCH, Zur Bedeutung von Schlaf und Traum 
songes allaient accompagner de façon presque structurale non seulement la vie et la mort du saint, mais aussi l'établissement et la vitalité de son culte.

La naissance du saint sera marquée souvent par un " rêve divinatoire de la mère enceinte ", dont un spécialiste a recueilli une centaine de témoignages. ${ }^{42} \mathrm{Il}$ est frappant de constater qu'un grand nombre de ces rêves trouve leur clé symbolique non dans la culture biblique, mais dans les cultures ethniques ambiantes (classique, celtique ou germanique) : un aigle qui vole sur le lit de la mère, la pleine lune qui tombe dans sa bouche, ou l'or rutilant qui sort de son sein. Ce qui frappe aussi est le fait que la signification du rêve n'est pas immédiate pour la mère. Elle lui est révélée par des experts ou des sages, ce qui rapproche et l'épisode et ses thèmes de l'oniromancie classique.

Cette importance du rêve n'est nullement limitée au moment de la naissance. On pourrait entreprendre des enquêtes analogues, qui produiraient une richesse analogue de matériaux, pour les autres moments importants de la biographie du saint : sa vocation, les décisions majeures, l'issue de ses projets, sa mort. Un exemple frappant en est la «grande vie » de saint Hugues, évêque de Lincoln (+ 1200) : non parce que ce personnage ait été particulièrement féru des rêves, mais parce que sa biographie, écrite par son secrétaire avec une extraordinaire richesse de détails, nous révèle mieux que d'autres le soin avec lequel les songes étaient retenus et racontés, l'empressement de procéder à leur interprétation, et le souci avec lequel on en conservait la mémoire, comme autant d'éléments essentiels d'une biographie. ${ }^{43}$ Un autre exemple de cette même richesse serait la vie de saint Bernard, illustrée (quant aux rêves) par ses écrits, et toute la littérature cistercienne primitive.

im Mittelalter. Krems, Medium Aevum Quotidianum, 1990, 400 p. (qui conclut à la vitalité des croyances oniriques, p. 341), et Raoul MANSELLI, "Il sogno come premonizione, consiglio e predizione nella tradizione medievale ", dans I sogni nel medioevo, p. 217-244.

42. Francesco LANZONI, "Il sogno presago della madre incinta nella letteratura medievale e antica ", Analecta Bollandiana, 45 (1927), p. 225-261. 43. Magna Vita S. Hogonis, éd. D.L DOUIE et D.H. FARMER, Londres, 1961 (puis 1985). 
Pour une biographie plus facile d'accès, on citera encore celle de François d'Assise. Le songe attribué au Pape Innocent III constitue une des pages les plus fameuses dans l'histoire de la papauté : «La basilique du Latran lui était apparue en songe prête à s'écrouler; et voici qu'un religieux chétif et minable s'en venait l'épauler pour en empêcher l'effondrement $»{ }^{44} \mathrm{La}$ Légende des trois compagnons ajoute le détail significatif sur l' "angoisse épistémologique » qui accompagne le rêve : "à son réveil, frappé de stupeur et d'effroi, le pape avait mis toute sa sagesse et sa perspicacité à découvrir ce que signifiait ce songe $»{ }^{45}$

Mais ce songe n'est que le plus fameux d'une douzaine de songes analogues que la tradition franciscaine nous a conservés. Tous comportent la même caractéristique : un récit élaboré, qui suppose une mémorisation tenace et soucieuse du détail ; l'importance existentielle du message transmis par le rêve ; l'interprétation du sens du message, par intuition ou par recours à un interprète autorisé. On recense ainsi le "songe de l'arbre élevé », qui préfigure la rencontre de François avec Innocent III $;^{46}$ le « songe des miettes rassemblées, que le saint raconte à tous ses compagnons, "désolé de n'en pouvoir percer le mystère ", et dont une voix du ciel viendra expliquer la signification $;{ }^{47}$ le « songe du palais des armes ", qui met en mouvement un apparat explicatif complexe (chez François et chez son biographe) $;^{48}$ le "songe du dialogue de Spolète " $; 9$ le "songe de la statue ", qui connut différentes rédactions et interprétations. ${ }^{50}$ À ce catalogue il faudrait ajouter le nombre

44. THOMAS DE CELANO, Vita secunda, 17 ; trad. VORREUX., p. 362. 45. Légende des trois compagnons, 51 ; trad. VORREUX, p. 838.

46. THOMAS DE CELANO, Vita Prima, 33 (trad. VORREUX, p. 245246) et Légende des trois compagnons, 53 (ibidem, p. 839).

47. THOMAS DE CELANO, Vita secunda, 209 (ibidem, p. 516) et BONAVENTURE, Legenda major, 4,11 (ibidem, p. 618-619).

48. THOMAS DE CELANO, Vita prima, 5 (ibidem, p. 218); Vita secunda, 5,6 (ibidem, pp. 349-351: version beaucoup plus élaborée); BONAVENTURE, Legenda major, I,3 (ibidem, p. 589-590).

49. THOMAS DE CELANO, Vita secunda, 6 (ibidem, p. 350); BONAVENTURE, Legenda major, I, 3 (ibidem, p.569-570) et Légende des trois compagnons, 6 (ibidem, p. 803).

50. THOMAS DE CELANO, Vita secunda, 82 (ibidem, p. 416-417, avec notes). 
extraordinaire d'épisodes qualifiés comme "visions ", mais dont le contexte ou la structure rédactionnelle laissent deviner qu'il s'agissait de visions par le rêve. Encore plus nombreux sont les rêves et les visions dont bénéficient les compagnons ou d'autres personnages de la vie de François. Cette place des songes chez François d'Assise n'est qu'un exemple, qu'on pourrait multiplier à loisir. Et relevons que pour introduire un de ces rêves, son biographe utilise la formule significative : "Le Seigneur l'accompagnait partout : ses révélations le laissaient tout joyeux et ses grâces lui rendaient courage. ${ }^{51}$

Mais il y a d'autres « lieux hagiographiques » dans lesquels le rêve prémonitoire ou prophétique intervient de façon décisive dans la structure des récits et des événements. Il en est ainsi dans ces milliers d'épisodes d'invention, élévation ou translation de reliques, qui sont à l'origine de tant de cultes. Le songe par lequel un évêque, un abbé ou un autre personnage important, se voit révéler l'emplacement oublié de ces trésors sacrés, avec la charge incontournable d'en établir ou raviver le culte, est une constante pratiquement absolue, depuis la découverte des corps de Gervais et Protais par l'évêque Ambroise de Milan jusqu'à la fin du Moyen Âge. ${ }^{52}$

Il en est encore ainsi des songes dans le culte des fidèles envers les saints. Le rêve est l'un des moyens privilégiés par lequel le saint suggère au fidèle de recourir à son pouvoir d'intercession. Il est encore le moyen par lequel, dans la pratique de l'incubatio, il annonce au fidèle que sa requête à été accordée et qu'il sera guéri. La pratique de l'incubatio comporte dans le nom même ("dormir dans ») la preuve de son lien avec le songe. Dans ses origines païennes, très bien connues par les textes et l'archéologie, elle consistait à dormir dans le sanctuaire thérapeutique, tant que la divinité tutélaire n'avait pas révélé par un songe la marche à suivre pour recouvrer la santé. Sous une forme moins ritualisée, la pratique de dormir dans le sanctuaire, aussi près que possible des reliques, et d'obtenir par une expérience onirique la garantie de la guérison, demeura vivace tout au long du Moyen Âge,

51. TOMAS DE CELANO, Vita Prima, 33 (trad. VORREUX, p. 245).

52. Voir Martin HEINZELMANN, Translationsberichte und andere Quellen des Reliquienkultes (Typologie des sources du Moyen Âge occidental), 33 ; Turnhout, 1979, et Pierre-André SIGAL, L'homme et le miracle dans la France médiévale (XI $-X I I^{e}$ s.), Paris, Cerf, 1985, 350 p. 
sous la pression populaire et contre les vues du clergé, qui aurait souhaité la fermeture nocturne des sanctuaires. Certains tombeaux comportaient des niches spéciales, aménagées sous le sarcophage du saint, justement pour permettre aux fidèles d'y passer la nuit. ${ }^{53}$

\section{Divination et présages dans le folklore pieux}

J'appelle "folklore pieux » un ensemble de pratiques et de croyances religieuses qui se situent en marge du christianisme officiel, ne faisant partie ni du droit, ni de la liturgie, ni de la théologie, ni de la spiritualité, ni de l'imagerie ou des valeurs fixées par la Bible ou la tradition patristique, mais qui en même temps jouissaient d'une large crédibilité, sinon d'une franche acceptation dans des textes et des milieux respectables de la société chrétienne. On en retrouve les traces dans les vies des saints, les histoires de fondation des ordres religieux, dans les histoires des sanctuaires et monastères, dans les traités sur les miracles et dans une vaste littérature de dévotion. Ce type de pratiques et de croyances dépasse certainement le niveau " populaire ", puisqu'il est accepté par des couches cultivées, sans toutefois jamais devenir une composante obligatoire du système chrétien. Un grand nombre de sacramentaux, de rituels et de grandes dévotions, comme celle du scapulaire ou des samedis de la Vierge, rentrent dans cette catégorie. Le "secret de Fatima » faisait encore partie, il y a quarante ans, de ces phénomènes à l'enracinement plus que " populaire ». L'extraordinaire catalogue de Merveilles du monde chrétien, compilé par un érudit italien à la fin du dix-septième siècle, demeure un bon miroir de ce que j'appelle ici « folklore pieux ». ${ }^{54}$

53. Voir, avec documents iconographiques, les témoignages cités dans Ronald C. FINUCANE, Miracles and Pilgrims. Popular Beliefs in Medieval England, London - Totowa, N.J. Rowman and Littlefield, 1977. Sur l'incubation dans l'histoire du christianisme il n'existe pas encore d'étude globale. Voir pour l'antiquité Mary HAMILTON, Incubatio or the cure of disease in pagan temples and christian churches,. St Andres - Londres, Henderson et Simkin, 1906, 223 p.

54. J. B. BAGATTA, Admiranda christiani orbis, quae (...) aut praeteritis extitere saeculis aut adhuc vigent (...). 2 vol., in-folio, plusieurs éditions, dont Augsbourg, 1700, que j'utilise ici. 
On ne sera pas surpris de constater la place que la prédiction de l'avenir occupe dans ces documents, sous forme de pressentiments, prophéties, prévisions, signes, présages, songes, visions. J'ai eu l'occasion d'évoquer, dans un travail antérieur, les thèmes divinatoires concernant la mort. ${ }^{55}$ Le premier thème est la connaissance anticipée de la date de la mort, ou de son imminence. Dans l'hagiographie, il s'agit d'un thème presque universel. À ce niveau hagiographique, il y a peu de traces d'une " technique ", fut-elle orthodoxe et pieuse, pour obtenir cette connaissance : elle est une certitude que le sujet trouve à l'intérieur de lui-même, ou le résultat d'une vision ou d'un songe. En dehors de l'hagiographie par contre, le thème devient plus articulé. Il me semble assuré que certaines formes de présages de la mort ont des racines folkloriques. Ainsi, le présage du cheval pleurant la mort prochaine de son maître, saint Columba, s'éclaire par la croyance très répandue dans le pouvoir mantique de cet animal.

Un thème enchaîné au premier est celui des "présages permanents » de la mort : dans une région, une famille ou une communauté religieuse, un événement d'un type donné signale à l'avance la mort d'un membre de ce couvent ou de cette famille. ${ }^{56}$ Ainsi à Messine, dans tel couvent de moniales, les volets qui couvrent une image miraculeuse de la Vierge s'ouvrent tout seuls chaque fois que l'une des moniales du monastère est appelée à mourir dans les jours suivants. Ainsi encore, "l'abbaye de Saint-Maurice d'Agaune, possède un vivier merveilleux, dans lequel on garde autant de poissons qu'il y a de religieux dans l'abbaye. Dès que l'un des religieux est frappé par une maladie, immédiatement l'un des poissons commence à flotter à la surface de l'eau. Si ledit religieux est destiné à mourir, le poisson mourra quelques jours avant lui, présage de ce qui arrivera au religieux ».

Un grande variété d'autres domaines se prêtent à une analyse semblable, qui donnerait de semblables résultats. Il y a le domaine des

55. «Le merveilleux autour de la mort dans le folklore pieux de l'Occident ", dans Essais sur la mort. Travaux d'un séminaire de recherche sur la mort..., éd. G. Couturier, A. Charron et G. Durand. Montréal, Fides, 1985, p. 299-334.

56. BAGATTA II, pp. 396-297 et, sous un chapitre plus général qui concerne « les présages permanents de choses tristes ", II, p. 167-168. 
objets perdus et retrouvés : des saints nombreux et des sanctuaires spécifiques sont spécialisés dans le recouvrement des objets perdus ou volés. Une rudimentaire technique divinatoire est souvent liée à ces dévotions, comme dans le cas de Saint Antoine de Padoue : "Ceux qui récitent le répons de Saint Antoine de Padoue, soit retrouvent immédiatement les objets perdus, soit arrivent à connaître de la façon la plus certaine dans quel endroit ils se trouvent, par qui ils ont été volés, et à quel usage on les emploie : ce que beaucoup de témoins dignes de foi affirment avoir toujours expérimenté dans leurs affaires ». Un autre vaste chapitre est celui des cloches qui sonnent sans intervention humaine, pour signaler un fait spécial, ou l'arrivée d'un saint inconnu, ou révéler des reliques oubliées, ou annoncer la mort d'un saint. Ici encore, beaucoup de ces prodiges se transforment en prodiges permanents, pour annoncer un malheur ou la mort d'un membre de la communauté, ou d'autres événements importants. ${ }^{57}$ Des moulins reconnaissent miraculeusement les blés obtenus par vol ou par rapine, et refusent de les moudre. D'autres objets, en tel ou tel autre endroit, manifestent le même pouvoir perpétuel de divination. ${ }^{58}$ Ajoutons le domaine encore plus considérable des animaux qui, par la voix, le mouvement ou d'autres façons, annoncent ou dévoilent l'avenir dans les circonstances les plus diverses : pour choisir ou désigner un candidat à l'épiscopat ou autre charge, pour signaler ou délimiter un lieu saint, pour tirer quelqu'un de l'embarras. ${ }^{59}$

\section{La tentation de la divination savante}

Un dernier domaine, enfin, vers lequel les intellectuels du Moyen Âge ont lorgné malgré les interdictions, autant et plus que le peuple, est celui des techniques divinatoires reliées aux astres, au calendrier et de ses parties, aux mouvements de la lune. La vaste synthèse de Lynn Thorndike me dispense d'entrer dans les détails d'une thématique qui a suscité une bibliographie abondante. ${ }^{60}$ Je n'évoquerai qu'en passant

57. BAGATTA, I, p. 344-353.

58. BAGATTA, I, p. 375 .

59. Très vaste secteur dans BAGATTA, I, p. 478-485. Toutes les sections sur chaque espèce d'animal (I, p. 413-470) comportent des épisodes à contenu divinatoire.

60. Voir Lynn THORNDIKE, A history of magic and experimental science during the first thirteen centuries of our era, New York et Londres, 
l'astrologie et ses composantes divinatoires, puisque ce domaine passait pour être celui de la science et était donc, en principe, acceptable. Les intellectuels les plus respectés considéraient comme une vérité axiomatique que les astres déterminaient tous les aspects du monde sublunaire, du sexe des embryons à la diffusion des épizooties, de l'issue des batailles à la fertilité des champs. Même Thomas d'Aquin a adhéré sans réticence à ce système, jusqu'à affirmer que, pour la grande majorité des gens, qui ne suivent que leurs instincts matériels, les corps célestes déterminent l'essentiel de leur vie. ${ }^{61}$

Mais j'évoquerai surtout ce monde en marge de l'astrologie qui, sur la base de traditions multiples (grecque, romaine, puis juive et arabe), de vagues principes théoriques (comme la correspondance entre les quatre éléments, les quatre saisons, les quatre humeurs, les quatre tempéraments, les quatre âges de la vie, les quatre points cardinaux, les quatre vents principaux, etc.) arrivaient à des formes pratiques de divination, utilisables même par les non savants. La forme la plus simple était le pronostic de l'année à partir du jour de la semaine dans lequel tombaient les kalendes de Janvier. Le tonnerre recevait une valeur divinatoire selon le jour de la semaine, le mois, ou d'autres variables de calendrier et de géographie. Les lunaria ou livres de la lune déterminaient l'avenir (l'issue d'une maladie, la réussite de tel type d'affaires, l'issue d'une bataille ou d'un combat individuel, par des formes diverses de spherae ou roues, à savoir des "roues de la fortune ", "roues de la vie et de la mort ", "roues de la maladie »), appliquaient des techniques plus complexes, utilisant le nom du patient. Les jours égyptiens étaient des listes de vingt-quatre jours (deux par mois) défavorables pour certaines entreprises, tandis que les jours arabes étaient des listes plus complexes, comportant jusqu'à une soixantaine de jours défavorables, et leur portée divinatoire respective.

Columbia Univ. Press, 1923 (réimpr., 1964), 2 voll., xlii-835 et 1036 p. L'œuvre de Thorndike confirme, avec des inépuisables catalogues d'oeuvres et de manuscrits, l'attrait de la divination pour une partie des intellectuels chrétiens. Mais voir en particulier I, p. 672-696.

61. Voir Thomas LITT, Les corps célestes dans l'univers de saint Thomas d'Aquin, Louvain-Paris, Nauwelaerts, 1963, p. 202, 240-241 [notamment le texte de De veritate 22, 9. ad 2]. 
Ce qu'on remarque, dans la littérature sur l'astrologie et la divination, est son lien avec les milieux officiels. "L'endroit le plus probable pour trouver, au Moyen Âge, des oeuvres d'astrologie et de divination sont les manuscrits qui contiennent des calendriers ecclésiastiques et des computs ", à savoir les monastères et les bibliothèques des cathédrales (L. Thorndike). Comme pour les sortes sanctorum, une partie au moins du clergé était donc compromise dans ces techniques qui, plus tard, seront folklorisées dans les prévisions des almanachs. Dans la même ligne, R. Kieckhefer a consacré un intéressant chapitre à " la nécromancie dans les bas-fonds cléricaux ». ${ }^{62}$

\section{Autres domaines du compromis}

J'arrête ici cette énumération des domaines dans lesquels l'Église médiévale a plus ou moins accepté, sous une forme ou l'autre, un certain usage de la divination. Cette énumération est partielle. D’autres domaines nous montreraient des formes tout aussi importantes d'ambivalence entre l'acceptation, le compromis et le refus. Chacun comporte une histoire et une bibliographie complexes.

Le plus important serait sans doute celui de l'ordalie, une réalité fondamentalement non biblique dans son concept et dans ses techniques ${ }^{63}$ - , qui fut toutefois acceptée et ritualisée par l'Église officielle jusqu'à la fin du XII ${ }^{\mathrm{e}}$ s., puis indirectement condamnée à partir du Concile Latran IV (1215). Elle survécut néanmoins, comme importante pratique populaire, jusqu'au XVII ${ }^{\mathrm{e}}{ }^{6}{ }^{64}$

Un autre domaine à explorer serait celui des prodiges et des présages. En général, la mentalité médiévale était portée, comme toute

62. KIECKHEFER, Richard, Magic in the middle ages, Cambridge, Cambridge University Press, 1989, p. 150-174 ( « Necromancy in the clerical underworld »).

63. Les anciens israëlites, comme beaucoup de leurs contemporains, étaient familiers avec des pratiques d'ordalie. On en mentionne, entres autres, dans $\mathrm{Nb}, 5,11,28$. Cependant, ce texte, à notre connaissance, ne semble pas avoir eu d'influence sur les pratiques médiévales.

64. Voir Robert BARTLETT, Trial by fire and water. The medieval judicial ordeal, Oxford, Clarendon Press, 1986, 182 p., et J.W. BALDWIN, "The intellectual preparation for the canon of 1215 against the ordeals ", Speculum, 36 (1961), p. 613-636. 
mentalité dite primitive, à donner beaucoup d'importance aux événements qui s'écartaient de l'ordinaire : comètes, éclipses, tremblements de terre, aurores boréales et autres phénomènes météorologiques. Chroniqueurs et historiens profanes donnaient en général une interprétation politique, économique ou sociale de ces événements. ${ }^{65}$ Mais l'Église ne tarda pas à construire une interprétation analogue des événements. Grégoire le Grand fut le premier théologien à développer une véritable hantise du sens religieux et " providentiel » des événements cosmologiques. Au niveau d'une pastorale plus fruste, cela se dégrada dans un providentialisme ingénu et immédiat, qui voyait partout des preuves de la bénédiction de Dieu pour les bons, et de la punition de Dieu pour les méchants.

Un dernier domaine de cette ambivalence serait probablement celui de la consultation des sorts (par des tables, dés, osselets). Nous ne savons pas jusqu'à quel point elle était véritablement utilisée. Mais reste le fait que, en même temps que l'Église condamnait durement tout recours à des techniques divinatoires dans un milieu et par du personnel indépendant de son contrôle (notamment dans les milieux populaires par des vetulae fatuae, "vieilles sottes »), elle semblait l'admettre dans un contexte et par un personnel religieusement qualifié. Telle est la position de Thomas d'Aquin, dans un article de sa Somme de théologie (II-II, q. 97, a. 8 : " Si la divination par les sorts est permise »), et dans un opuscule plus élaboré Sur les sorts. Dans les deux cas on énumère une série de situations dans lesquelles la consultation par les sorts n'est pas permise, mais on finit par admettre une utilisation partielle, dans un système plutôt moral que technique : "Dans un cas de nécessité, et avec le respect qui s’impose, il est permis d'implorer par les sorts la décision divine ».

\section{Conclusion}

Ma conclusion ne pourra être qu'un constat. En refusant aussi bien de "laïciser " radicalement la divination, que de créer une " divination chrétienne » institutionnalisée et ouverte à tous, la pastorale médiévale s'est placée dans la position intenable de proposer un barrage de défenses, sans offrir d'alternatives valables. Elle a dû

65. Voir Paul ROUSSET, "Le sens du merveilleux à l'époque féodale ", $L e$ Moyen Âge, 62 (1956), p. 25-37. 
accepter elle-même des compromis étendus avec des pratiques divinatoires trop enracinées, et trop importantes dans l'équilibre social, pour être totalement abolies.

Mais par cette attitude ambivalente, l'Église a réussi à garder, pour tout ce qui concerne la divination et ses techniques, un droit fondamental de regard et d'interprétation. Comme pour l'authentification du miracle et de la prophétie, elle a réussi - sans doute bona fide - à arracher au fidèle ordinaire un outil d'autonomie dans la gestion de sa vie, aussi bien religieuse que profane. Malgré les apparences, l'histoire de la divination dans le christianisme médiéval n'est pas l'histoire de sa répression et de son élimination, mais celle de sa soumission progressive au contrôle de l'autorité et de l'idéologie dominante.

\section{RÉSUMÉ}

Le christianisme condamna dès les premiers siècles, surtout après SaintAugustin, toute forme de divination : l'oniromancie, la bibliomancie et toutes autres techniques traditionnelles, qui semblaient trop entachées de paganisme. Dans la pratique, toutefois, l'Église fut beaucoup plus tolérante, lorsque ces mêmes pratiques étaient utilisées dans un contexte qu'elle considérait sans danger théologique ou pastoral. Le recours à la divination devenait ainsi un outil discrétionnaire de contrôle, notamment par rapport à la divination populaire. Par un choix très différent, l'Islam considéra les techniques divinatoires comme une branche des sciences profanes, qui ne menaçaient en rien la religion et que ses fidèles pouvaient pratiquer librement.

\section{ABSTRACT}

From the earliest period, and especially after St. Augustine, Christianity condemned all forms of divination: oniromancy, bibliomancy and the other traditional techniques which seemed tainted with paganism. Nevertheless, the Church was considerably more tolerant in practice when the same techniques were used in a context without evident theological risk or danger to the pastorate. Thus, divination became an instrument of control, to be used at Church's discretion, especially in relation to popular customs. Islam followed a completely different course. It considered divinatory techniques as a branch of secular science, which did not threaten religion and which the faithful could freely practice. 